Research Article

\title{
Numerical Simulation on the Whole Sinking Process of Open Caisson with an Improved SPH Method
}

\author{
Jiahe Zhang $\mathbb{D}$ \\ College of Water Conservancy and Hydropower Engineering, Hohai University, Nanjing 210098, China \\ Correspondence should be addressed to Jiahe Zhang; zhangjh_hhu@163.com
}

Received 23 December 2020; Revised 25 January 2021; Accepted 17 February 2021; Published 25 February 2021

Academic Editor: Erivelton Geraldo Nepomuceno

Copyright (c) 2021 Jiahe Zhang. This is an open access article distributed under the Creative Commons Attribution License, which permits unrestricted use, distribution, and reproduction in any medium, provided the original work is properly cited.

\begin{abstract}
The phenomena of dynamic change in the material interfaces and mechanical properties are often involved in the caisson construction. Using conventional methods to simulate these phenomena is quite difficult due to the extremely large deformation. In this study, we proposed an improved soil-water-caisson interaction algorithm with the method of smoothed-particle hydrodynamics (SPH). This algorithm dealt with the support domain truncation of the particles near the blade and applied $\delta-\mathrm{SPH}$ to avoid the pressure fluctuation. Meanwhile, the application of dynamic particles birth and death method could simulate the whole sinking process of an open caisson with underwater soil excavation. According to the comparison between SPH simulation and centrifuge test, the distribution of sidewall effective soil pressure was consistent, which indicated promising applicability of the algorithm. It should be noted that the considerable excess pore water pressure appeared in the surrounding soil under the blade. With the dissipation of the pressure over time, the effective soil stress increased correspondingly, and it would lead to the increasing difficulty of the sinking process. Therefore, the caisson should be avoided to stop for a long time during the sinking process or it would cause the stagnation of sinking. This algorithm could simulate engineering problems involving underwater construction effectively and provide theoretical and technical support for underwater excavation, shield tunneling, and other engineering problems.
\end{abstract}

\section{Introduction}

Open caisson plays a significant role in deep foundation, and it is widely used in harbor breakwaters and large-span bridges due to its advantages of strong integrity, high stiffness, large bearing capacity, and convenient construction [1-4].

In recent years, many studies have investigated open caissons. Allenby et al. [5] concluded with a series of practical guidelines for construction through four successful open caisson sinking operations. Hao et al. [6] applied GPS RTK techniques to monitor the construction of super-deep-water open caisson timely. Lenzi et al. [7] developed the "guided caisson technique" to prevent the conventionally occurring pendulousness of the open caisson. Zhao et al. [8] declared that soil arching and rotation of principal stress were the important mechanisms which affected the mechanical behaviors of open caissons. Chavda et al. [9] investigated the penetration resistance which was affected by varying tapered angles of the blade edge through a series of model tests, and a number of studies concerned about the spatial stress state of the cutting edge $[10,11]$. BIM technology was used to assist monitoring of open caisson construction of super-large bridge [12]. Pan et al. [13] summarized the sinking resistance changing regularity and distribution characteristics of large caisson foundation and discussed the construction impact on the surrounding environment. In a word, with the rapid development of infrastructure construction, open caissons play an increasingly important role in construction.

The research on resistance to side friction has always been focused during the whole sinking process of the open caisson, and it is also the basis for the design of caisson structure and the measure of auxiliary sinking. Because the calculation of the resistance in engineering design depends on strong experience, its calculation result is often quite different from the actual situation. Therefore, the research on related experiments and numerical simulation is 
essential. In terms of model tests, Wang et al. [14] invented a new type of microfriction sensor to measure the sidewall friction directly and obtained the variation tendency of sidewall friction during the open caisson sinking process. Jiang et al. [15] designed the model test on the background of largest open caisson and established a calculation model to describe the behavior of the migration. Zhou et al. [16] implemented the sinking simulation of the open caisson through the centrifuge model test, and the state of actual construction was well simulated due to centrifugal field which could compensate for the loss of stress because of model size reduction. Nevertheless, the dynamical excavation process could not be simulated accurately because the experimental depth was not continuous.

In the aspect of numerical simulation, Jiang et al. [17] analyzed the impact on the excavation of the open caisson sinking based on the finite element (FEM) numerical simulation. $\mathrm{Li}$ et al [18] built a three-dimensional soil-structure interaction FEM modeling and analyzed the influence of the caisson structure diameter on the internal force in the condition of first sinking. Lai et al. [19] simulated the construction of giant deep-buried circular open caisson in undrained clay by the Coupled Eulerian-Lagrangian (CEL) approach. Although this method could avoid mesh distortion to a certain extent, it also caused calculation failure when dealing with the problem of moving material interfaces (e.g., soil-water-caisson interface). Due to the extremely large deformation caused by the cutting action of soil, underwater excavation, and soil flow during the sinking process, it is difficult to implement numerical simulations using conventional methods (e.g., finite element method) because of mesh distortion and winding, which will lead to calculation failure. Consequently, the scope of application is restricted because of the necessary prerequisite for extra special technologies. However, smoothed-particle hydrodynamic (SPH) is a Lagrangian numerical method, which can solve problems such as large deformation and interface migration of different materials.

SPH was initially proposed to deal with astrophysical problems [20, 21]. After that, Violeau and Issa [22] applied SPH into complex free surface flow problems. Drucker-Prager (D-P) model was introduced into the SPH method [23], which makes SPH widely used in the geotechnical engineering field. A fluid-solid coupling SPH algorithm was established to simulate the large deformation problem of complex soil and water interaction based on the two-phase mixture theory [24-27]. Wen et al. [28] used an improved weakly compressible SPH mixture model and investigated the effects of reef permeability on the spatial distributions of wave-induced current inside and outside the inhomogeneous coral reef body. Khayyer et al. [29] proposed a mixture model based on the incompressible SPH and presented a numerical wave flume with porous media of variable porosity. In a word, SPH has been widely used in various fields of research. However, there are few studies on the application of SPH in the simulation of caisson construction. Wang et al. [30] used single-phase soil particles to simulate the sinking process of an open caisson with the SPH method, but it has limited applicability because of the difficulty to reflect the situation of wading engineering construction veritably. Although the modeling took into account the large deformation of soil, the complex soil-water coupling was not considered, and then the change rule of pore water pressure (PWP) could not be obtained. In fact, the generation and dissipation of excess pore water pressure (EPWP) has an important effect on steady sinking of the caisson and benefits to improving the calculation accuracy of soil-water-caisson coupling. It also has a practical value to guide the design and construction of open caissons. Therefore, it is necessary to consider the complex soil-watercaisson coupling interaction in the simulation of an open caisson construction.

In this paper, a coupled soil-water-caisson interaction algorithm was presented based on previous studies of two-phase coupling SPH algorithm. The influence of support domain truncation of the particles near the blade had been concerned, and $\delta-\mathrm{SPH}$ was applied to avoid the pressure fluctuation caused by density oscillation. Then, accurate distribution of PWP could be obtained. Simultaneously, the technique of dynamic particles birth and death was used to realize underwater soil excavation, and the complex coupling interaction of soil-water-caisson could be effectively simulated during the whole sinking process.

\section{Theoretical Basis}

2.1. The Model of Soil-Water Mixture. Each phase is assumed to occupy different volume fractions in the macroscopic mixture by the two-phase mixture theory [31-33] when considering the saturated soil-water mixture. The mass conservation equations are given by

$$
\begin{aligned}
& \frac{\mathrm{d} \rho_{s}}{\mathrm{~d} t}=-\rho_{s} \nabla \cdot v_{s}, \quad \rho_{s}=(1-n) \widetilde{\rho}_{s}, \\
& \frac{\mathrm{d} \rho_{f}}{\mathrm{~d} t}=-\rho_{f} \nabla \cdot v_{f}, \quad \rho_{f}=n \widetilde{\rho}_{f},
\end{aligned}
$$

where subscripts $s$ and $f$ represent soil and water, respectively; $v$ is the spatially averaged velocity; $\rho$ is the apparent density; $\widetilde{\rho}_{s}$ is the particle density of soil; $\widetilde{\rho}_{f}$ is the intrinsic density of water; and $n$ is the volume fraction of water, namely, the porosity.

Assume that the compression of soil is completely caused by the deformation of the soil skeleton and soil particles are incompressible, that is, to say, $\widetilde{\rho}_{s}$ is constant. Then, the governing equation of the soil porosity can be given by

$$
\frac{\mathrm{d} n}{\mathrm{~d} t}=-(1-n) \nabla \cdot v_{s} .
$$

The total stress tensor of the mixture is decomposed into equation (4) based on the principle of Terzaghi's effective stress [34]:

$$
\sigma=\sigma^{\prime}-(1-n) \widetilde{p}_{f} I+n \widetilde{\sigma}_{f}, \quad \widetilde{\sigma}_{f}=-\widetilde{p}_{f} I+\tilde{\tau}_{f},
$$

where $\sigma$ and $\sigma^{\prime}$ are, respectively, the total stress tensor and the effective stress; $\widetilde{p}_{f}$ denotes the PWP; and $\widetilde{\tau}_{f}$ represents the shear stress of water.

The momentum equations of the mixture can also be written as 


$$
\begin{aligned}
\rho_{s} \frac{\mathrm{d} v_{s}}{\mathrm{~d} t} & =\nabla \cdot \sigma^{\prime}-(1-n) \nabla \cdot \tilde{p}_{f}+f_{d}+\rho_{s} g \\
\rho_{f} \frac{\mathrm{d} v_{f}}{\mathrm{~d} t} & =-\nabla \cdot\left(n \tilde{p}_{f}\right)+\nabla \cdot\left(n \tilde{\tau}_{f}\right)+\widetilde{p}_{f} \nabla \cdot n-f_{d}+\rho_{f} g \\
f_{d} & =\frac{n^{2} \widetilde{\rho}_{f} \mathcal{g}\left(v_{f}-v_{s}\right)}{k},
\end{aligned}
$$

where $\mathrm{f}_{d}$ denotes the viscous drag force derived from the Darcy law; $k$ is the hydraulic conductivity; and $g$ is the gravity acceleration.

2.2. Constitutive Model for Soil and Water. In this study, we used the Drucker-Prager (D-P) constitutive model [23] to describe the soil behavior:

$$
\begin{aligned}
\dot{\sigma}^{\alpha \beta}= & \sigma^{\alpha \gamma} \dot{\omega}^{\beta \gamma}+\sigma^{\beta \gamma} \dot{\omega}^{\alpha \gamma}+2 G \dot{e}^{\alpha \beta} \\
& +K \dot{\varepsilon}^{\gamma \gamma} \delta^{\alpha \beta}-\dot{\lambda}\left[3 K \alpha_{2} \delta^{\alpha \beta}+\frac{G}{\sqrt{J_{2}}} s^{\alpha \beta}\right], \\
\dot{\lambda}= & \frac{3 \alpha_{1} K \dot{\varepsilon}^{\gamma \gamma}+\left(G / \sqrt{J_{2}}\right) s^{\alpha \beta} \dot{\varepsilon}^{\alpha \beta}}{9 \alpha_{1} \alpha_{2} K+G},
\end{aligned}
$$

where $\alpha, \beta$, and $\gamma$ represent Cartesian components; $\delta$ denotes Kronecker's delta; $G$ denotes shear modulus; $K$ denotes bulk modulus; $\dot{\varepsilon}^{\alpha \beta}$ and $\dot{\omega}^{\alpha \beta}$ are, respectively, the total strain rate and rotation rate tensor; $\dot{e}^{\alpha \beta}$ and $s^{\alpha \beta}$ represent the tensor of deviatoric strain rate and deviatoric stress, respectively; $\dot{\lambda}$ is the plastic multiplicative factor; $\alpha_{1}$ is the Drucker-Prager constant related to the friction angle $\phi$; and $\alpha_{2}$ is the dilatancy factor affected by the dilation angle $\psi$.

Meanwhile, the water phase was regarded as a Newtonian fluid, and the shear stress $\tilde{\tau}^{\alpha \beta}$ is calculated by the dynamic viscosity $\mu$ and the shear strain rate $\varepsilon_{f}^{\alpha \beta}$ of the water.

$$
\begin{aligned}
\tilde{\tau}_{f}^{\alpha \beta} & =\mu \varepsilon_{f}^{\alpha \beta} \\
\varepsilon_{f}^{\alpha \beta} & =\frac{\partial v_{f}^{\alpha}}{\partial x^{\beta}}+\frac{\partial v_{f}^{\beta}}{\partial x^{\alpha}}-\frac{2}{3}\left(\nabla \cdot v_{f}\right) \delta^{\alpha \beta} \\
\tilde{p}_{f} & =B\left[\left(\frac{\widetilde{\rho}_{f}}{\tilde{\rho}_{f 0}}\right)^{\gamma}-1\right],
\end{aligned}
$$

where $B$ is a problem-dependent parameter that sets a limit to the maximum density variation; $\gamma$ is a constant normally set to seven; and $\widetilde{\rho}_{f 0}$ is the reference true density of water.

2.3. $\mathrm{SPH}$ Implementation. In $\mathrm{SPH}$, a limited number of particles are used to discretize the computational domain, and field variables and material properties are assigned to the particles [35]. The integral interpolation of the field variables is given as follows:

$$
\langle f(x)\rangle=\int_{\Omega} f\left(x^{\prime}\right) W\left(x-x^{\prime}, h\right) \mathrm{d} x^{\prime}
$$

where $W$ is the kernel or smoothing function and $h$ is the smoothing length, which defines the influence domain $\Omega$ of $W$.

Then, the conservation equations of soil and water could be rewritten in the SPH form by using SPH particle approximation. For simplicity, the subscripts $i, j$ and $a, b$ are used to identify the particles of soil and water.

Firstly, the governing equation of the soil porosity can be rewritten as follows by SPH particle approximation, and the porosity of water particle a can be obtained by interpolation of its neighboring soil particles:

$$
\begin{aligned}
\frac{\mathrm{d} n_{i}}{\mathrm{~d} t} & =\left(1-n_{i}\right) \sum_{j=1}^{N} \frac{m_{j}}{\rho_{j}} v_{j i} \cdot \nabla_{i} W_{i j}, \\
n_{a} & =\sum_{i=1}^{N} \frac{m_{i}}{\rho_{i}} n_{i} W_{a i},
\end{aligned}
$$

where $v_{j i}=v_{j}-v_{i}$.

Since the assumption considered above is that the compression of soil is completely caused by the deformation of the soil skeleton, soil particles are incompressible. The apparent density of soil can be obtained through the following equation. In addition, the continuity equation of water also can be rewritten as follows:

$$
\begin{aligned}
\rho_{i} & =\left(1-n_{i}\right) \widetilde{\rho}_{s}, \\
\frac{\mathrm{d} \rho_{a}}{\mathrm{~d} t} & =-\rho_{a} \frac{1}{\rho_{a}} \sum_{b=1}^{M} m_{b}\left[v_{b}-v_{a}\right] \cdot \nabla_{a} W_{a b}=\sum_{b=1}^{M} m_{b} v_{a b} \cdot \nabla_{a} W_{a b} .
\end{aligned}
$$

Similarly, the momentum conservation equations also can be rewritten as

$$
\begin{aligned}
\frac{\mathrm{d} v_{i}}{\mathrm{~d} t}= & \sum_{j=1}^{N} m_{j}\left(\frac{\sigma_{i}^{\prime}}{\rho_{i}^{2}}+\frac{\sigma_{j}^{\prime}}{\rho_{j}^{2}}-\Pi_{i j}\right) \cdot \nabla_{i} W_{i j} \\
& -\left(1-n_{i}\right) \sum_{a=1}^{M} \frac{m_{a}}{\rho_{a}} \frac{\tilde{p}_{a}}{\rho_{i}} \nabla_{i} W_{a i} \\
& +\sum_{a=1}^{M} \frac{m_{a}}{\rho_{a}} \frac{f_{i a}}{\rho_{i}} W_{a i}+g_{i}, \\
\frac{\mathrm{d} v_{a}}{\mathrm{~d} t}= & -\sum_{b=1}^{M} m_{b}\left(\frac{\tilde{p}_{a} n_{a}}{\rho_{a}^{2}}+\frac{\tilde{p}_{b} n_{b}}{\rho_{b}^{2}}-\Pi_{a b}\right) \cdot \nabla_{a} W_{a b} \\
& +\sum_{b=1}^{M} m_{b}\left(\frac{\tilde{\tau}_{a} n_{a}}{\rho_{a}^{2}}+\frac{\tilde{\tau}_{b} n_{b}}{\rho_{b}^{2}}\right) \cdot \nabla_{a} W_{a b} \\
& +\sum_{i=1}^{N} \frac{m_{i}}{\rho_{i}} \frac{\widetilde{p}_{a}}{\rho_{a}} n_{i} \nabla_{a} W_{a i}-\sum_{i=1}^{N} \frac{m_{i}}{\rho_{i}} \frac{f_{i a}}{\rho_{a}} W_{a i}+g_{a} .
\end{aligned}
$$

In equations (18) and (19), $\Pi_{i j}$ and $\Pi_{a b}$ denote artificial viscosity terms [36] which are used to prevent the unphysical 
penetration between particles. It is worth noting that there is usually another method to calculate the momentum equations of water particles based on the pressure gradient formulation. More details about the so-called ISPH can be found in [37].

2.4. Boundary Contact Treatment. When dealing with the interaction between the caisson and soil/water particles, a frictional sliding contact algorithm proposed by Wang et al. [38] is adopted. This algorithm assumes that the partial penetration is allowed, and the contact force can be obtained by

$$
\begin{aligned}
& F_{n}=(1-\varsigma)\left[\frac{2 m_{i}}{(\Delta t)^{2}}\left(d_{0}+G \cdot n\right)\right] n, \\
& F_{\tau}^{\prime}=\frac{2 m_{i}}{(\Delta t)^{2}} \Delta u_{\tau}, \\
& F_{\tau}= \begin{cases}\frac{\xi\left|F_{n}\right|}{\left|F_{\tau}^{\prime}\right|} F_{\tau}^{\prime}, \quad \text { if }\left|F_{\tau}^{\prime}\right|>\xi\left|F_{n}\right|, \\
F_{\tau}^{\prime}, \quad \text { otherwise, }\end{cases}
\end{aligned}
$$

where $F_{n}$ and $F_{\tau}$ are the contact force, respectively, along the normal and tangential direction of structure surface, and it should be noted that $F_{\tau}=0$ when the water particles contact the surface of the structure; $\varsigma$ defines the extent of the residual penetration and is set as $0.01 \sim 0.1$; and $\xi$ is the frictional coefficient. More details can be obtained by the mentioned reference.

\subsection{Boundary Particle Support Domain Truncation and $\delta-$} SPH Applications. During the sinking process of an open caisson, the support domain of soil/water particles is truncated by the blade, and the treatment for this problem is shown in Figure 1. Meanwhile, the density field of those boundary particles is initialized every time step through equation (23). When equation (12) is used to obtain the PWP, the pressure oscillations easily occurs due to density fluctuation. To eliminate the pressure oscillations during the sinking process of the open caisson, the $\delta$-SPHmethod [39-41] is applied in this study. Then, more accurate distribution of the PWP can be obtained, and equation (17) can be rewritten as follows:

$$
\begin{aligned}
\rho_{a} & =\frac{\sum_{b=1}^{M} m_{b} W_{a b}}{\sum_{b=1}^{M} m_{b} / \rho_{b} W_{a b}} \\
\frac{\mathrm{d} \rho_{a}}{\mathrm{~d} t} & =\sum_{b=1}^{M} m_{b} v_{a b} \cdot \nabla_{a} W_{a b}+\delta_{f} h_{a} c_{f} \sum_{b=1}^{M} \frac{m_{b}}{\rho_{b}} \Psi_{a b} \cdot \nabla_{a} W_{a b} \\
\Psi_{a b} & =2\left(\rho_{a}-\rho_{b}\right) \frac{x_{a b}}{\left|x_{a b}\right|^{2}}
\end{aligned}
$$

where $v_{a b}=v_{a}-v_{b}, \delta_{f}$ is a constant normally set to 0.1 and $c_{f}$ is the sound speed of water.

\section{Numerical Simulation and Analysis}

3.1. Control Measures of Soil Excavation and Water Replenishment. Due to the excavation of underwater soil during the construction of the caisson, rapid drawdown of the inside groundwater level produces the seepage, which could cause the large area of peripheral soil to collapse. To avoid the risk of the instability of the caisson, water replenishment measures are usually taken to ensure the stable groundwater level. As Figure 2 shows, the technique of dynamic particles birth and death is used to realize measures of soil excavation and water replenishment. To simplify the calculation, the number of water particles is not changed before and after excavation. The newly generated water particles replace the removed water particles in the original position and should conform to the following properties:

$$
\begin{aligned}
\frac{m_{a}^{\prime}}{m_{a}} & =\frac{n_{a}^{\prime}}{n_{a}}, n_{a}^{\prime}=1.0, \\
m_{a}^{\prime} v_{a}^{\prime} & =m_{a} v_{a}, \\
\widetilde{p}_{a}^{\prime} & =\widetilde{p}_{a} ; \widetilde{p}_{a}^{\prime}=\widetilde{p}_{a}, \widetilde{\tau}_{a}^{\prime}=\widetilde{\tau}_{a},
\end{aligned}
$$

where the superscript 'denotes the newly generated water particles. It is worth noting that removing soil results in the change of the volume fraction of water in the macroscopic mixture. Assuming that the velocity of the macrosupplementary water could be ignored, equation (26) gives the momentum conservation equation before and after excavation.

3.2. Establishment of the Numerical Model. As of concern, there are no relevant tests or measurement data for continuous sinking and excavation of an open caisson in the whole process, and the study of sidewall friction is always key and difficult points. Therefore, the centrifuge test [16] was used as a controlled trial to verify the effectiveness of the soilwater-caisson interaction algorithm in this section. The centrifuge test was carried out under the condition of $90 \mathrm{~g}$ by TLJ-2 geotechnical centrifuge at Southwest Jiaotong University. The equivalent sinking depth is $36 \mathrm{~m}$, and the tilt angle of the blade is 45 . The same parameters of the test were used for modeling which is shown in Table 1. For simplicity, the problem was conducted under two-dimensional plain strain conditions, and half of the open caisson was used for building the model. In addition, the caisson was treated as rigid structure, and segmental construction of the caisson was ignored. The sketch of the open caisson sinking model is shown in Figure 3. The coefficient of friction between the soil and the caisson structure is set as 0.472 .

The two phases were severally discretized into 2700 particles. Initially, soil particles and water particles were overlapped and then moved, respectively, with their own governing equations. Ghost particles were used to deal with the boundaries. And, the right boundary corresponded to a free-slip boundary condition (I), meaning that the particles could move without restraints along the boundary. The left 


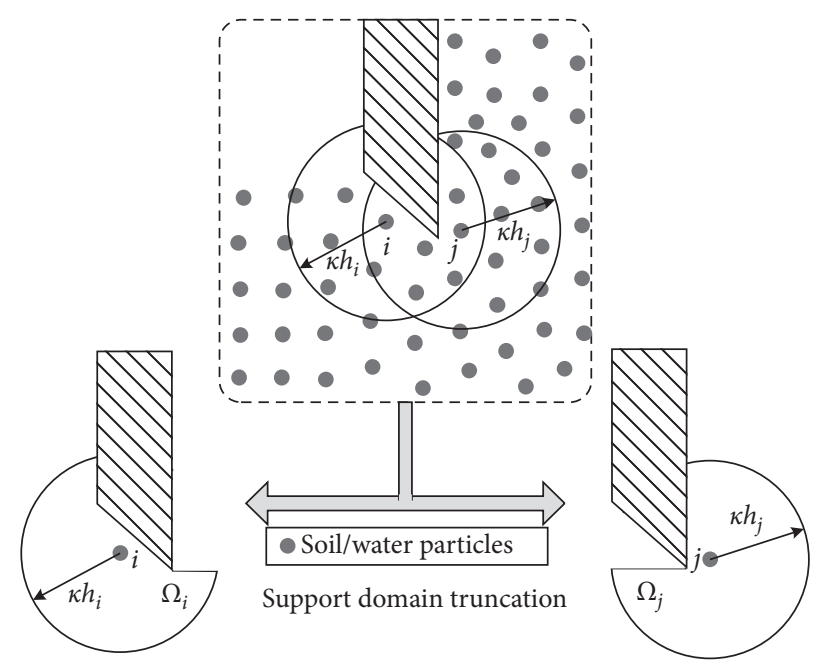

FIGURE 1: The schematic diagram of particle support domain truncation processing.

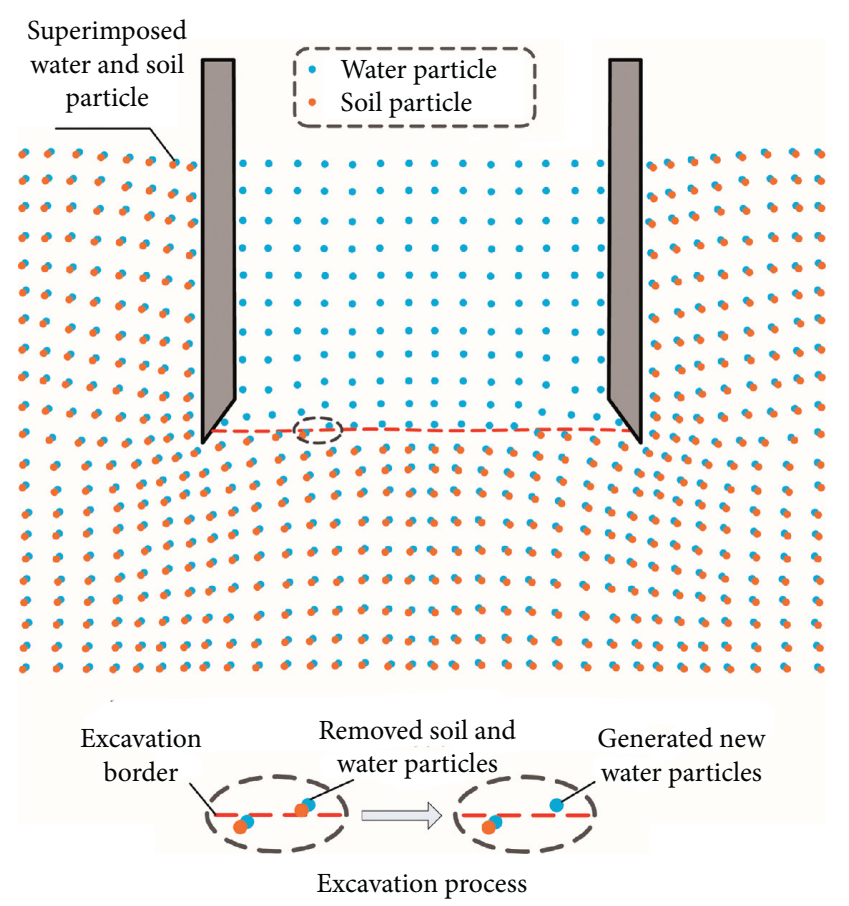

FIGURE 2: Schematic diagram of excavation process.

and bottom boundaries corresponded to nonslip boundary conditions (II). Assume that the soil was completely saturated and the free surface of water was equal to the soil. The effective stress distribution of the soil under the initial condition was estimated according to effective gravity and the lateral pressure coefficients. In this study, the sinking speed of the open caisson was set as $1 \mathrm{~m} / \mathrm{s}$, and the time step $\Delta t$ was set to $1.0 \times 10^{-6} \mathrm{~s}$.

3.3. Analysis of Numerical Simulation Result. Figure 4 shows the sidewall effective soil pressure distribution comparing between SPH simulation and the centrifuge test when the caisson sinks to the specified depth $(27 \mathrm{~m}$ and $36 \mathrm{~m})$. It is easy to obtain that there are similar regularities of the distribution of
TABLe 1: Material properties and dimension of model.

\begin{tabular}{lcc}
\hline Soil properties & Water parameters & Model dimension \\
\hline$\tilde{\rho}_{s}=2704 \mathrm{~kg} / \mathrm{m}^{3}$ & $\mu=0.001 \mathrm{~Pa} . \mathrm{s}$ & $H=108 \mathrm{~m}$ \\
$E_{S}=45 \mathrm{MPa}$ & $\tilde{\rho}_{f}=1000 \mathrm{~kg} / \mathrm{m}^{3}$ & $W=144 \mathrm{~m}$ \\
$k=1.0 \times 10^{-5} \mathrm{~m} / \mathrm{s}$ & $c_{f}=30.0 \mathrm{~m} / \mathrm{s}$ & $L=86.4 \mathrm{~m}$ \\
$n=0.422$ & & $D=3.6 \mathrm{~m}$ \\
$c=0 \mathrm{kPa}$ & & $\theta=45^{\circ}$ \\
$\phi=36.2^{\circ}$ & \\
$v=0.3$ & \\
\hline
\end{tabular}

the pressure, which increase gradually at the beginning and decrease gradually after reaching the peak value at a certain depth. The main reason is that the height of soil in the well is lower than that outside of the well when the caisson has reached a certain depth. The soil pressure difference is appeared inside and outside the blade, which makes the soil outside the well flow inwards and forms a pressure relaxation zone, thus causing the effective soil pressure on the sidewall to increase first and then decrease with the depth. It should be noted that the centrifuge test cannot simulate the dynamic process of sinking and excavation of the caisson, and a specific buried depth should be selected to carry out the test by applying transitory centrifugal acceleration. However, the soil pressure relaxation zone outside the caisson moves down continuously with the simulation of the whole process of continuous sinking and excavation by the SPH method. The effective soil pressure on the sidewall is a continuous release process; therefore, the slope of the pressure distribution curve simulated by the $\mathrm{SPH}$ method is lower than the centrifuge test, and the peak value is also smaller. Meanwhile, the position of the peak value is closer to the blade. Comprehensive analysis shows that the SPH simulation of the effective soil pressure on the sidewall is basically consistent with the centrifuge test, which indicates the feasibility and effectiveness of the SPH soil-water-caisson coupling algorithm in the simulation of the whole sinking and excavation process.

During the whole sinking process of the open caisson, accurate distribution of the PWP (left) and that of the EPWP (right) can be obtained simply. Figure 5 gives the pressure 


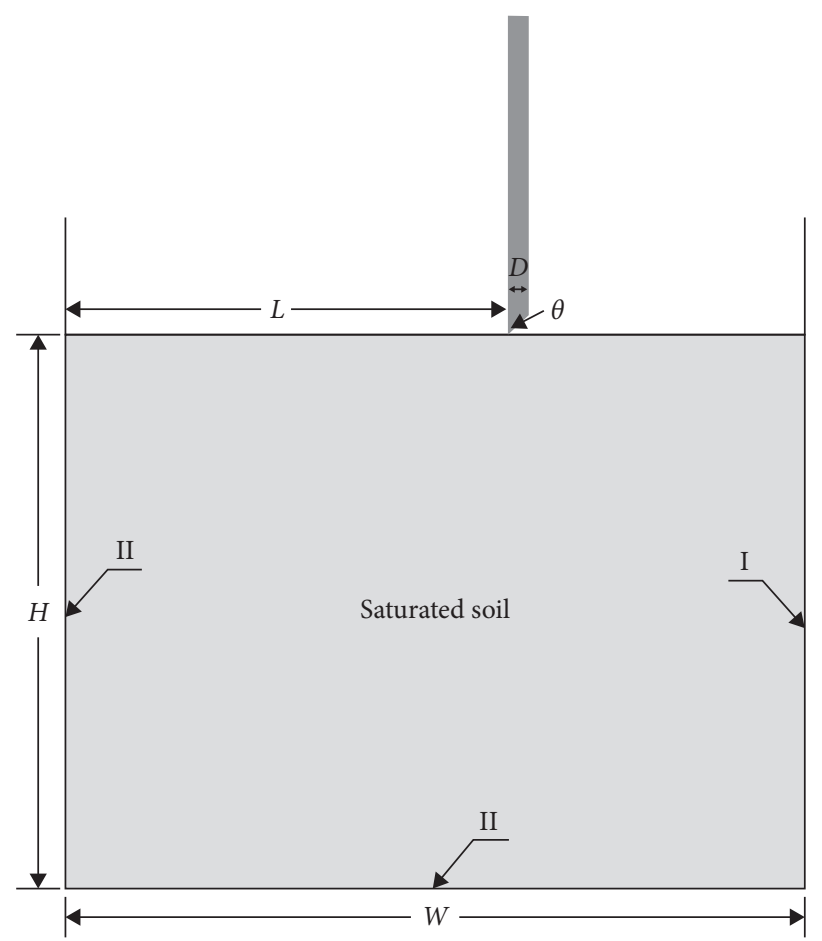

FIGURE 3: Sketch of the open caisson sinking model.

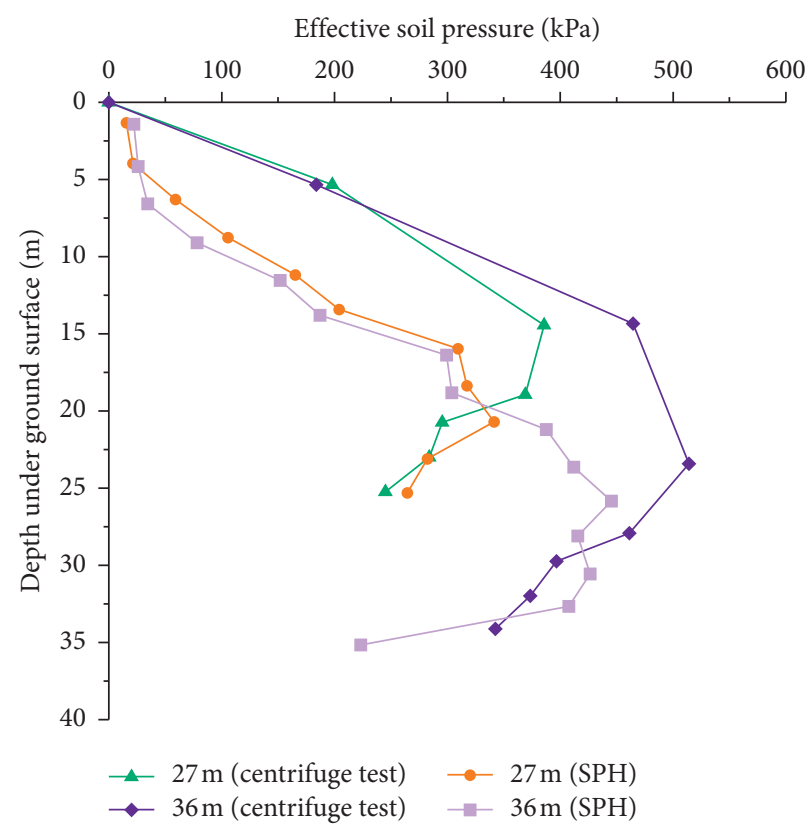

FIGURE 4: Distribution of the effective soil pressure acting on the sidewall.

distribution with two special depths, one is the blade immersed in the soil without excavation and the other is the maximum depth. When $d=7 \mathrm{~m}$, the PWP far away from the caisson wall was not affected that was similar to the distribution of hydrostatic pressure, but it increased obviously near the blade edge. According to the right side of Figure 5(a), the soil under the blade generated $200-240 \mathrm{kPa}$ EPWP and decayed along the direction away from the cutting edge gradually. As shown in Figure 5(b), the caisson reached the maximum sinking depth that was $36 \mathrm{~m}$. The distribution of PWP had a significant change especially the soil under the blade, the EPWP increased significantly which exceeded $500 \mathrm{kPa}$, and elliptic high pressure distribution indicated that the attenuation rate along the normal direction of the blade bevel was obviously weaker than other directions. It should be concerned that the surrounding soil under the blade occurred great deformation and generated considerable EPWP during the construction of the open caisson. Furthermore, the EPWP would dissipate over time, the 


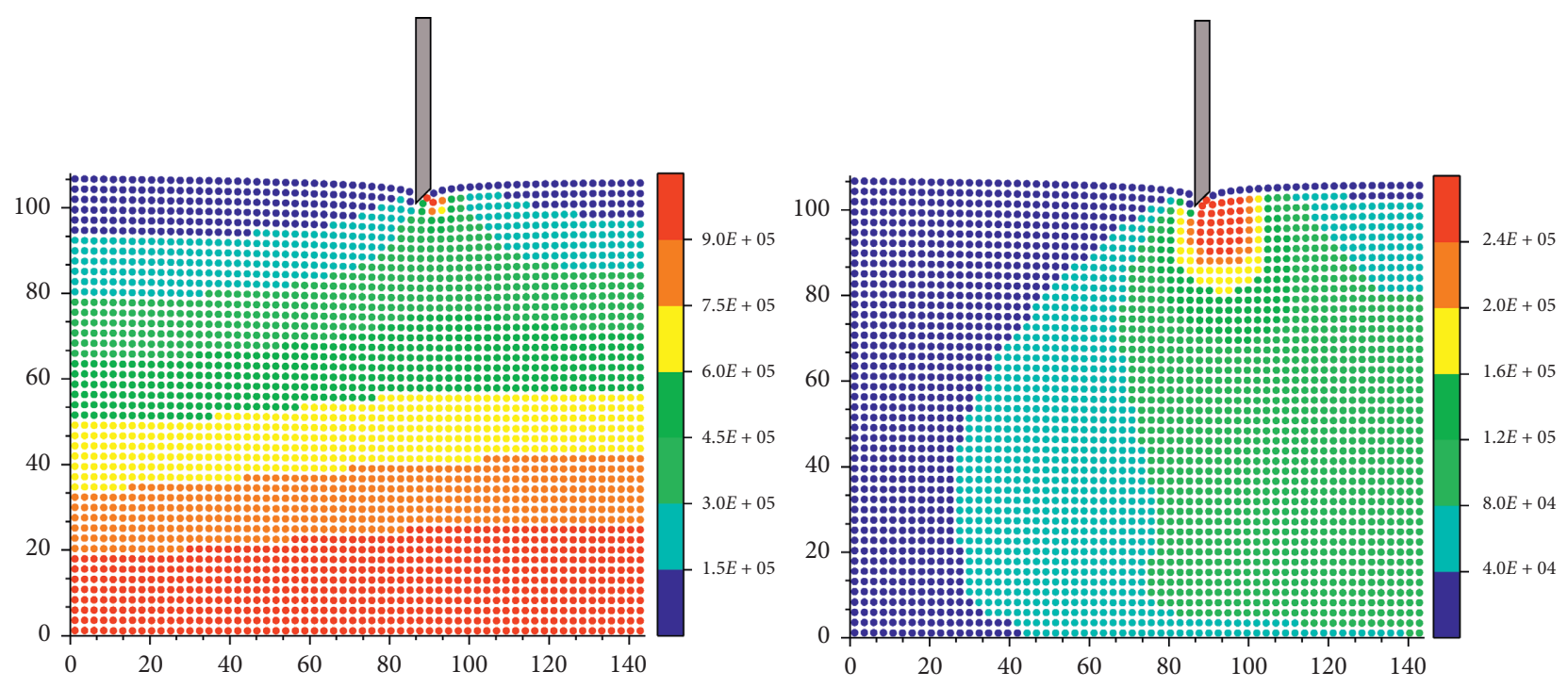

(a)
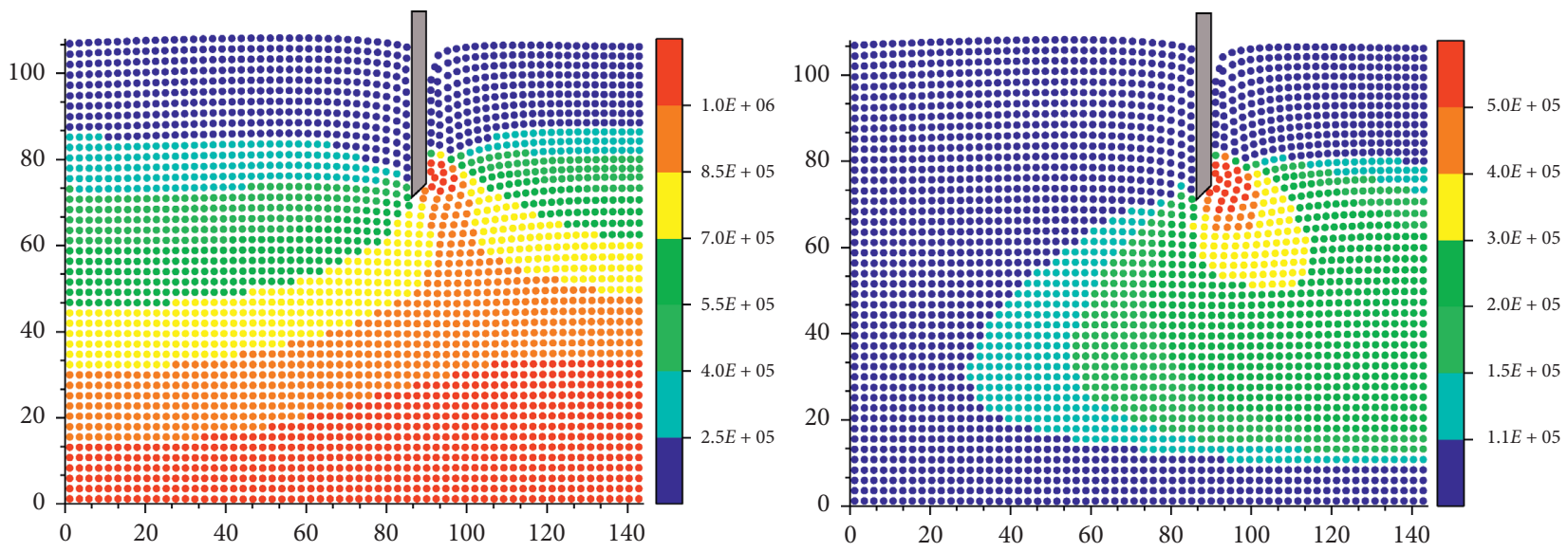

(b)

FIgURE 5: Distribution of PWP (left) and EPWP (right) of the open caisson during the sinking process: (a) $d=7 \mathrm{~m}$ and (b) $d=36 \mathrm{~m}$.

effective stress of the soil would increase on the basis of the principle of Terzaghi's effective stress, and the sinking resistance would also increase accordingly. Therefore, it is preferable for the caisson to sink continuously and slowly. Except the needs of the construction (e.g., segmented splicing construction of the caisson), it should be avoided to stop for a long time during the sinking process or it would cause the stagnation of sinking.

\section{Discussion}

Due to the meshless nature, the SPH method could solve large deformation problems in the fields of hydraulic engineering, geotechnical engineering, ocean engineering, and so on. This paper is aimed to broaden the application of SPH to the construction of wading engineering. Based on previous studies of the two-phase coupling SPH method, a coupled soil-watercaisson interaction algorithm was presented to realize the simulation of the whole sinking and excavation process of the open caisson. It can veritably and effectively simulate the large deformation of soil-water-caisson interaction and realize measures of soil excavation and water replenishment, and it can also be significant for the design, construction guidance, and reduction of safety hazard of the similar wading engineering, and the results and prospects are obtained as follows:

(1) By comparing SPH simulation of the effective soil pressure on the sidewall with centrifuge test, it can be obtained that they have the similar regularity of the distribution of the pressure. Due to the difference in height of soil inside and outside the caisson, a pressure relaxation zone is formed outside the caisson, which results in the trend of the pressure distribution increasing first and then decreasing with depth. It indicates the feasibility and effectiveness of the soil-water-caisson coupling algorithm.

(2) During the sinking process of the caisson, the surrounding soil under the blade generates considerable EPWP. It means the effective stress of 
the soil would increase as the EPWP dissipates over time, and the sinking resistance would also increase accordingly. Therefore, in the process of construction, the caisson should be avoided to stop for a long time or it would cause the stagnation of sinking.

(3) In this study, the time step depends on the permeability of soil to maintain the stability of the calculation, the smaller time step seriously increased the computational time cost, and the proposed algorithm has certain requirements for particle resolution. The authors believe that this algorithm can be improved by applying the GPU parallel computation and particle refinement method.

\section{Data Availability}

The data used to support the findings of this study are included within the article.

\section{Conflicts of Interest}

The authors declare that there are no conflicts of interest regarding the publication of this paper.

\section{Acknowledgments}

This research was supported by the National Natural Science Foundation of China (grant no. 51779084).

\section{References}

[1] S. K. Chakrabarti, P. Chakrabarti, and M. S. Krishna, "Design, construction, and installation of a floating caisson used as a bridge pier," Journal of Waterway, Port, Coastal, and Ocean Engineering, vol. 132, no. 3, pp. 143-156, 2006.

[2] F. E. Lefler and V. D. R. Romero, "Reinforced concrete caissons for port structures in Spain," in Proceedings of ICE: Maritime Engineering, vol. 162, pp. 73-81, 2009.

[3] T. Matsuda, K. Maeda, M. Miyake, J. Miyamoto, H. Sumida, and $\mathrm{K}$. Tsurugasaki, "Instability of a caisson-type breakwater induced by an earthquake-tsunami event," International Journal of Geomechanics, vol. 16, no. 5, p. C4016003, 2016.

[4] B. D. Rogers, R. A. Dalrymple, and P. K. Stansby, "Simulation of caisson breakwater movement using 2-D SPH," Journal of Hydraulic Research, vol. 48, no. 1, pp. 135-141, 2010.

[5] D. Allenby, G. Waley, and D. Kilburn, "Examples of open caisson sinking in Scotland," in Proceedings of the Institution of Civil Engineers-Geotechnical Engineering, vol. 162, no. 1, pp. 59-70, 2009.

[6] Y. G. Hao, X. L. Liu, and J. B. Liu, "GPS RTK techniques for construction monitoring of super deep-water open-caisson," Applied Mechanics and Materials, vol. 580-583, pp. 28652871, 2014.

[7] M. Lenzi, W. Halsegger, and S. Semprich, "Experimental and numerical investigations of the guided caisson method," in Proceeding of the the 16th International Conference on Soil Mechanics and Geotechnical Engineering, pp. 1625-1628, Osaka, Japan, September 2005.

[8] X. Zhao, J. Xu, B. Mu, and B. Li, "Macro- and meso-scale mechanical behavior of caissons during sinking," Journal of Testing and Evaluation, vol. 43, pp. 363-375, 2015.
[9] J. T. Chavda, S. Mishra, and G. R. Dodagoudar, "Experimental evaluation of ultimate bearing capacity of the cutting edge of an open caisson," International Journal of Physical Modelling in Geotechnics, vol. 20, pp. 1-14, 2020.

[10] G.-G. Yea and T.-H. Kim, "Vertical cutting edge forces measured during the sinking of pneumatic caisson," Marine Georesources \& Geotechnology, vol. 30, no. 2, pp. 103-121, 2012.

[11] F. Y. Yan, Y. C. Guo, and S. Q. Liu, "The bearing capacity analyses of soil beneath the blade of circular cassion," Advanced Materials Research, vol. 250-253, pp. 1794-1797, 2011.

[12] S. Zhu, H. Nan, and Y. Zheng, "Research and application of BIM cloud monitoring system for open caisson construction of super large bridge," IOP Conference Series: Earth and Environmental Science, vol. 510, p. 052077, 2020.

[13] Y. Pan, C. Wang, and F. Liang, "Sinking resistance distribution characteristics of large open caissons and related engineering problems," Structural Engineers, vol. 36, pp. 134-143, 2020.

[14] J. Wang, Y. Liu, and Y. Zhang, "Model test on sidewall friction of open caisson," Rock and Soil Mechanics, vol. 34, pp. 659666, 2013.

[15] B.-N. Jiang, M.-T. Wang, T. Chen, L.-L. Zhang, and J.-L. Ma, "Experimental study on the migration regularity of sand outside a large, deep-water, open caisson during sinking," Ocean Engineering, vol. 193, p. 106601, 2019.

[16] H. X. Zhou, J. L. Ma, K. Zhang, C. Y. Luo, and B. Yang, "Study on sinking resistance of large and deep caisson based on centrifugal model test," Rock and Soil Mechanics, vol. 40, pp. 3969-3976, 2019.

[17] B. Jiang, J. Ma, and J. Chu, "The influence of soil surrounding the caisson cutting edge to excavation and sinking," in Proceeding of the International Conference on Geotechnical and Earthquake Engineering, pp. 435-448, Chongqing, China, October 2018.

[18] P. Li, E. Song, and T. Zheng, "Initial sinking method for large open caisson in a highway bridge project," in Proceeding of the China-Europe Conference on Geotechnical Engineering, pp. 1692-1696, Vienna, Austria, August 2018.

[19] F. Lai, S. Liu, Y. Deng, Y. Sun, K. Wu, and H. Liu, "Numerical investigations of the installation process of giant deep-buried circular open caissons in undrained clay," Computers and Geotechnics, vol. 118, p. 103322, 2020.

[20] L. B. Lucy, "A numerical approach to the testing of the fission hypothesis," The Astronomical Journal, vol. 82, pp. 1013-1024, 1977.

[21] R. A. Gingold and J. J. Monaghan, "Smoothed particle hydrodynamics: theory and application to non-spherical stars," Monthly Notices of the Royal Astronomical Society, vol. 181, no. 3, pp. 375-389, 1977.

[22] D. Violeau and R. Issa, "Numerical modelling of complex turbulent free-surface flows with the SPH method: an overview," International Journal for Numerical Methods in Fluids, vol. 53, no. 2, pp. 277-304, 2007.

[23] H. H. Bui, R. Fukagawa, K. Sako, and S. Ohno, "Lagrangian meshfree particles method (SPH) for large deformation and failure flows of geomaterial using elastic-plastic soil constitutive model," International Journal for Numerical and Analytical Methods in Geomechanics, vol. 32, no. 12, pp. 1537-1570, 2008.

[24] Y. Huang, W. Zhang, Z. Dai, and Q. Xu, "Numerical simulation of flow processes in liquefied soils using a soil-watercoupled smoothed particle hydrodynamics method," Natural Hazards, vol. 69, no. 1, pp. 809-827, 2013. 
[25] C. Wang, Y. Wang, C. Peng, and X. Meng, "Smoothed particle hydrodynamics simulation of water-soil mixture flows(Article)," Journal of Hydraulic Engineering, vol. 142, Article ID 04016032, 2016.

[26] H. H. Bui and G. D. Nguyen, "A coupled fluid-solid SPH approach to modelling flow through deformable porous media," International Journal of Solids and Structures, vol. 125, pp. 244-264, 2017.

[27] H. Wu, J. Wang, C. Wang, and J. H. Wang, "Soil-water-structure interaction algorithm in smoothed particle hydrodynamics (SPH) with application to deep-penetrating problems," International Journal of Computational Methods, vol. 17, p. 1850135, 2018.

[28] H. Wen, B. Ren, P. Dong, and G. Zhu, "Numerical analysis of wave-induced current within the inhomogeneous coral reef using a refined SPH model," Coastal Engineering, vol. 156, p. 103616, 2020.

[29] A. Khayyer, H. Gotoh, Y. Shimizu, K. Gotoh, H. Falahaty, and S. Shao, "Development of a projection-based SPH method for numerical wave flume with porous media of variable porosity," Coastal Engineering, vol. 140, pp. 1-22, 2018.

[30] J. Wang, H. Hua, and W. P. Lian, "Penetration simulation for an open caisson using mesh-free SPH method," in Proceeding of the Computer Methods and Recent Advances in Geomechanics, pp. 105-109, Oka, Japan, September 2014.

[31] D. A. Drew, "Mathematical modeling of two-phase flow," Annual Review of Fluid Mechanics, vol. 15, no. 1, pp. 261-291, 1983.

[32] Y. Wang and K. Hutter, "A constitutive model of multiphase mixtures and its application in shearing flows of saturated solid-fluid mixtures," Granular Matter, vol. 1, no. 4, pp. 163-181, 1999a.

[33] Y. Wang and K. Hutter, "A constitutive theory of fluid-saturated granular materials and its application in gravitational flows," Rheologica Acta, vol. 38, no. 3, pp. 214-223, 1999 b.

[34] K. Terzaghi, Theoretical Soil Mechanics, Wiley, New York, NY, USA, 1943.

[35] M. B. Liu, G. R. Liu, and Z. Zong, "An overview on smoothed particle hydrodynamics," International Journal of Computational Methods, vol. 5, no. 1, pp. 135-188, 2008.

[36] J. J. Monaghan, "Simulating free surface flows with SPH," Journal of Computational Physics, vol. 110, no. 2, pp. 399-406, 1994.

[37] X. Zheng, S. Shao, A. Khayyer, W. Duan, Q. Ma, and K. Liao, "Corrected first-order derivative ISPH in water wave simulations," Coastal Engineering Journal, vol. 59, no. 1, p. 1750010, 2017.

[38] J. Wang, H. Wu, C. Gu, and H. Hua, "Simulating frictional contact in smoothed particle hydrodynamics," Science China Technological Sciences, vol. 56, no. 7, pp. 1779-1789, 2013.

[39] M. Antuono, A. Colagrossi, S. Marrone, and D. Molteni, "Free-surface flows solved by means of SPH schemes with numerical diffusive terms," Computer Physics Communications, vol. 181, no. 3, pp. 532-549, 2010.

[40] S. Marrone, M. Antuono, A. Colagrossi, G. Colicchio, D. Le Touzé, and G. Graziani, " $\delta$-SPH model for simulating violent impact flows," Computer Methods in Applied Mechanics and Engineering, vol. 200, no. 13-16, pp. 1526-1542, 2011.

[41] M. Antuono, A. Colagrossi, and S. Marrone, "Numerical diffusive terms in weakly-compressible SPH schemes," Computer Physics Communications, vol. 183, no. 12, pp. 2570-2580, 2012. 